\title{
Dalla crisi allo sviluppo sostenibile. Il ruolo dei sistemi di misurazione e controllo
}

\author{
Luciano Marchi*
}

\begin{abstract}
Moving from crisis to sustainable development. The role of control systems

This article offers some reflections and insights to interpret the economic impact of the current pandemic crisis, and similar crises that could arise in the future, and the conditions for recreating sustainable development.

Firstly, to move from evaluating crisis to generating economic value and sustainability requires a new corporate governance approach for interacting with the social and environmental context, integrating risk management and performance management (Marchi, 2019; Greco, D’Onza, 2020). Sustainability can be reinforced by adopting a new theory of value created for all stakeholders, included suppliers, customers, employees, the territory and the social community, but also for the environment and for the company itself, ensuring adequate remunerations of the resources and conditions of lasting economic equilibrium of companies (Giannessi, 1960).

To guarantee a long-term economic balance together with social, environmental and corporate sustainability, the role of measurement and control systems is fundamental. In this regard, the following aspects can be highlighted (Marchi, Paolini, 2020): 1) integrated accounting and budgeting systems must be developed with an income perspective; 2) excessive emphasis on specific performance indicators, especially financial ones, must be avoided, in order to pass to a "systemic reading" of the set of indicators at an economic-social level; 3 ) a circular choice and an adequate remuneration of the production factors in the supply network must be guaranteed in order to increase the value created internally in the network, with the contribution of strategic suppliers who must be partners of the company; 4) finally, a "culture of sustainability" must be implemented in the personnel planning, control and incentive systems (integrating financial indicators with sustainability indicators) and not only in the reporting systems for the outside.
\end{abstract}

Keywords: value, value creation, sustainability, control systems, financial indicators, sustainability indicators

\footnotetext{
*Università di Pisa, E-mail: luciano.marchi@unipi.it; luciano48.marchi@gmail.com.
}

Management Control (ISSN 2239-0391, ISSNe 2239-4397), 2020, 3

DOI: 10.3280/MACO2020-003001 


\section{Creazione di valore e sviluppo sostenibile}

Sul tema della creazione di valore affrontato negli ultimi editoriali della rivista Management Control (Marchi, 2019; Greco, D’Onza, 2020) si impongono alcune riflessioni e approfondimenti a seguito della crisi pandemica attuale e di analoghe crisi che potrebbero presentarsi in futuro. Particolare attenzione deve essere rivolta alla definizione di sistemi e soluzioni per fronteggiare le situazioni di crisi e per governare i processi di sviluppo aziendale, nel rispetto delle condizioni di equilibrio economico durevole, anche in termini di sostenibilità sociale e ambientale.

Per fronteggiare la crisi e creare processi di sviluppo sostenibile, è necessario realizzare un approccio integrato di governo aziendale, in stretto rapporto con il contesto ambientale, anche al fine di individuare e coordinare i punti di incontro, gestire l'innovazione e aumentare le opportunità di valore condiviso.

Dobbiamo anche imparare a reagire in modo sempre più rapido, mettendo in comune le conoscenze, utilizzando le nuove tecnologie dell'informazione e della comunicazione, con il ricorso a modelli di supporto decisionale, sviluppando capacità di verifica e interpretazione diretta dei risultati, nella loro capacità segnaletica, senza delegare l'interpretazione dei dati ad algoritmi di calcolo (Varanini, 2020).

La sintesi interpretativa in termini di valore (a livello economico-finanziario) è possibile solo accogliendo, nell'ambito del sistema politico-istituzionale, una nuova teoria del valore creato per l'insieme degli stakeholder e la comunità sociale, ma anche per l'ambiente e per l'azienda in sé, per garantire la conservazione e la congrua remunerazione delle risorse (strutturali, intellettuali, umane, relazionali, sociali e ambientali) e le condizioni di equilibrio economico durevole delle aziende (Giannessi, 1960).

Se si privilegia l'ottica di breve termine dei mercati finanziari, se non vengono garantiti congrui accantonamenti per fronteggiare sia i rischi specifici che il rischio generale d'azienda, le condizioni di equilibrio economico durevole potrebbero essere compromesse dalle crisi che, come quella attuale, potrebbero perdurare per più anni.

Se la sostenibilità non rientra tra gli obiettivi strategici del governo aziendale, analogamente, le condizioni di equilibrio economico rischiano di non essere durevolmente garantite. Quello qui accolto, infatti, è un concetto di sostenibilità ampio, tale da contemplare la ricerca della sostenibilità dell'ambiente omnicomprensivo e dell'azienda stessa. 


\section{Approccio integrato di governo aziendale}

La sostenibilità passa attraverso un approccio integrato di gestione dei rischi e delle performance, con l'obiettivo di creare valore per l'insieme degli stakeholders: per gli azionisti, per gli altri finanziatori, per i fornitori, per i clienti, per i lavoratori dipendenti, per il territorio e per la comunità sociale, ma anche per l'ambiente e per l'azienda in sé, sempre nella prospettiva di un equilibrio economico durevole.

Per quanto riguarda, invece, la coerenza tra l'obiettivo della sostenibilità e quello della creazione di valore, il dubbio è che l'obiettivo di creare valore per gli azionisti non sia del tutto coerente con l'obiettivo di creare valore per l'azienda in sé nella rete cooperativa con i fornitori e con i dipendenti e che l'obiettivo di creare valore per i clienti non sia coerente con l'obiettivo di creare valore per la comunità sociale.

La coerenza può essere creata facendo riferimento all'economicità sociale (Matacena, 1999) e al "bene dell'impresa" come "distinto e sovraordinato rispetto al bene di questo o quello dei suoi stakeholder" (Coda, 2012, p. 75), per arrivare a una nuova teoria del valore che non privilegi gli interessi degli investitori, ma sia basata sulla riaffermazione dell'azienda come sistema che sia capace di raccordare la sostenibilità con le condizioni di equilibrio economico durevole.

Sul ruolo del mercato e dell'intervento pubblico in tema di sostenibilità, utilizziamo le parole di Milton Friedman, "la dottrina della responsabilità sociale comporta che siano i meccanismi politici, non quelli di mercato, a determinare l'allocazione di risorse scarse per scopi alternativi" (Friedman, 1970, pp. 1-2). L'interpretazione qui accolta, tuttavia, non è quella fornita da Friedman (che la responsabilità sociale delle imprese "è solo quella di produrre profitti"), piuttosto che senza l'intervento diretto dello Stato in campo economico, l'ottica del profitto può distruggere le risorse naturali e creare squilibri sociali.

Più in generale, condividiamo il pensiero di Bernard Maris "il sistema economico deve reggersi su tre gambe: un terzo di mercato, un terzo di pubblico, un terzo di economia sociale (cooperative, volontariato, ecc.)". Volere imporre il "solo mercato" è pernicioso come il "tutto Stato". E soprattutto, i mercati, specie i mercati finanziari, devono restare un gioco a somma zero, cioè un gioco dove chi entra, se vince e guadagna, guadagni a spese degli altri che sono entrati nel mercato, non del settore pubblico, del denaro dei contribuenti, e dell'economia sociale, come oggi avviene" (Maris, 2000, pp. 62-67). 
Quindi da un sistema oggi centrato sui mercati finanziari, il futuro modello di sviluppo economico dovrà essere sempre più centrato sull'economia reale e sulla sostenibilità (entrambe orientate alla "circolarità), riportando al centro del sistema l'azienda come unità economica elementare, con la sua capacità di creare valore (equilibrio economico durevole) mediante la competizione e la cooperazione tra aziende diverse.

La responsabilità sociale, pertanto, deve essere riferita ai comportamenti di tutti i soggetti coinvolti nel sistema economico, nel quadro della regolamentazione pubblica, ma anche, necessariamente, all'intervento diretto dello Stato in campo economico. Pertanto, l'intervento pubblico dovrebbe realizzarsi su due piani strettamente collegati: garantire, in un mondo sempre più globalizzato, che siano i meccanismi politici, e non quelli di mercato, a determinare l'allocazione di risorse scarse per scopi alternativi; determinare l'integrazione della responsabilità sociale verso l'esterno con quella verso l'interno, cioè verso l'azienda in sé, dunque garantire le condizioni di esistenza delle singole unità produttive (Catturi, 1997, p. 50).

Se un'azienda sceglie un business sostenibile capace di creare ricchezza riesce anche a diffondere innovazione e vantaggi per tutta la società. I punti di incontro tra sistema azienda e sistema ambiente devono essere individuati e coordinati al fine di aumentare le opportunità di valore condiviso. La sostenibilità è diventata quindi una variabile strategica primaria nella definizione delle scelte di business.

Se le aziende adottassero un approccio strategico trattando i temi connessi alla responsabilità sociale come parte integrante dei processi decisionali, riuscirebbero a sfruttare economicamente i vantaggi relativi alle logiche sociali (quindi anche a quelle ambientali). In tale ambito, rientrano le strategie relativi all'economia circolare, mediante la definizione di un sistema di approvvigionamento-produzione-vendita ancorato a ri-alimentare continuamente il circuito economico e la progettazione di prodotti/servizi che derivino da "materie" non prime ma seconde, ovvero di facile (corta) reperibilità, ovvero anch'esse progettate con criteri di sostenibilità (Marchi, Paolini, 2020). Anche le fasi del ciclo di lavorazione dovrebbero essere progettate con la ricerca costante di innovazioni tecnologiche così che il processo risulti meno impattante rispetto all'ambiente; la progettazione sostenibile non risparmia le fasi distributive (con la logistica trasporti nella riflessione centrale) ma soprattutto la progettazione deve considerare, per i beni strumentali, la riparazione, la fattibilità della rigenerazione e del riutilizzo e, nel caso di beni di consumo, della gestione dei rifiuti e del loro riciclaggio (Paolini, 2018, p. 7). 


\section{Evoluzione dei sistemi di controllo interno-esterno}

Ulteriori riflessioni si impongono sull'evoluzione dei sistemi di controllo: essi assumono una sempre maggiore proiezione esterna, a tutela degli interessi delle diverse classi di stakeholders. Più in generale, si ritiene che i sistemi di controllo interno debbano necessariamente integrare la dimensione economica e quella sociale, non solo nelle aziende pubbliche, ma anche nelle aziende private, a prescindere dalla loro finalità non-profit o profit. La suddetta riflessione scaturisce dalle finalità che tutte le aziende - pubbliche e private (profit e non-profit) - dovrebbero perseguire (Paolini, 2018, p. 14): 1) un equilibrio economico a valere nel tempo; 2) il soddisfacimento dei bisogni umani.

A tale riguardo, anche alla luce dei cambiamenti in atto nei sistemi economici, nella prospettiva della cosiddetta "economicità-sociale" (Matacena, 1993), si assiste ad un sempre maggiore avvicinamento tra aziende pubbliche e private:

- nelle aziende pubbliche, la finalità prioritaria del soddisfacimento dei bisogni umani è direttamente perseguita integrando, nei sistemi di controllo interno gestionale, la dimensione "individuale" e quella "plurale" o sociale del valore pubblico riconosciuto (Borgonovi, Mussari, 2011, p. 111);

- nelle aziende private, un sempre più diffuso controllo sociale spinge anche le aziende con finalità profit a perseguire il consenso della comunità sociale ed il soddisfacimento, indiretto, dei bisogni umani (Bertini, 1990, p. 23 ss).

In conclusione, l'evoluzione dei sistemi di pianificazione, controllo e reporting determina una sempre maggiore integrazione tra i diversi livelli e tra le diverse prospettive interne-esterne, nella dimensione economico-finanziaria e in quella economico-sociale. In un tale quadro di integrazione, si ritiene utile sottolineare le seguenti auspicabili linee evolutive (Marchi, Paolini, 2020):

1) devono essere sviluppati sistemi contabili e di bilancio integrati nell'ottica reddituale-patrimoniale;

2) si deve evitare l'eccessiva enfasi su specifici indicatori di performance, specie quelli finanziari. Senza una "lettura sistemica" del complesso degli indicatori a livello economico-sociale, si rischia di: a) confondere il 
mezzo (risultati finanziari) con il fine (equilibrio economico-sociale a valere nel tempo); b) avere una visione di breve termine (la cosiddetta miopia manageriale);

3) deve essere garantita una scelta circolare e una congrua remunerazione dei fattori produttivi, nella rete di fornitura, al fine di consolidare il capitale strutturale, il capitale umano e il capitale relazionale. Il valore aggiunto aziendale, lo ripetiamo, è il valore creato internamente alla rete, con il contributo dei fornitori strategici che devono essere partner dell'azienda per realizzare assieme l'equilibrio economico-sociale a valere nel tempo;

4) si deve sottolineare che, per quanto concerne la sostenibilità, non vi è solo la tutela del territorio dal punto di vista economico, sociale e ambientale rispetto alle attività aziendali, ma anche la salvaguardia delle condizioni di esistenza di ciascuna singola azienda come produttrice di ricchezza;

5) deve realizzarsi, infine, una "cultura della sostenibilità" nei sistemi di pianificazione, controllo e incentivazione del personale (integrando indicatori finanziari con indici di sostenibilità/riduzione dei rischi legati alla sostenibilità) e non solo nei sistemi di reporting per l'esterno.

In relazione al punto sub 3. va aggiunto che nei rapporti di fornitura all' interno della rete, specie per i servizi ad alto contenuto di lavoro (professionisti esterni di varia tipologia), l'obiettivo prioritario è quello di aumentare il valore creato e ripartirlo nel rispetto degli accordi di cooperazione. Nelle fasi di start-up o di crisi aziendale, inoltre, capita spesso che il compenso della prestazione sia molto più basso del valore creato, o corrisponda addirittura solo al rimborso delle spese sostenute (al fine di sostenere l'equilibrio economico dell'azienda che riceve la prestazione) e ciò va tenuto nella giusta considerazione.

\section{Teoria del valore: dall'ottica patrimoniale all'ottica reddituale nella prospettiva economico-sociale}

Per quanto riguarda la teoria del valore, riconoscendo il contributo che hanno offerto (e possono tuttora offrire) gli studi sul valore economico del capitale e sulla catena del valore di Porter (1985), riteniamo che si debba prioritariamente far riferimento ai flussi economico-reddituali nel legame con le operazioni di gestione. L'assunto di base è che l'azienda è un sistema nel quale tutte le operazioni di gestione e tutte le connesse relazioni con gli stakeholder creano (o distruggono) valore: 
- il valore creato dall'azienda è espresso dal valore aggiunto nei processi produttivi intesi in senso lato, e viene "riconosciuto" nelle relazioni con i clienti in termini di ricavo delle vendite e delle prestazioni di servizi ma anche nelle relazioni con gli utenti dei servizi pubblici. In questo secondo caso, il "riconoscimento" del valore creato è espresso direttamente, in termini di ricavo, in presenza di un mercato, o indirettamente, in termini di provento (contributo o trasferimento), da parte della rappresentanza "politica" degli utenti ${ }^{1}$;

- al valore creato si contrappone il valore attribuito, cioè distribuito, ai vari stakeholders nelle diverse fasi dei processi produttivi e finanziari. Il prezzo pagato per le risorse acquisite, sia quelle utilizzate sia quelle da utilizzare, dunque il costo di acquisto, è la misura di tale valore.

Riteniamo inoltre che il bilancio debba esprimere le misure sia del valore creato che di quello distribuito a favore dei diversi destinatari, attuali e potenziali, economici e sociali, con una logica integrata reddituale-patrimoniale. Non come adesso, laddove il bilancio privilegia gli interessi degli investitori, dunque dei mercati finanziari (Ronen, 2008; Andrei, 2008). Perché è il reddito che determina il valore del capitale se il capitale è investito in processi di produzione reale; viceversa è il capitale che determina il reddito se esso confluisce negli investimenti finanziari. Solo in questi casi, come per i gestori dei fondi di investimento, potrebbe essere giustificato il ricorso al fair value "finanziario".

La sintesi economico-reddituale, ai fini della rendicontazione sociale, dovrebbe essere incentrata sul valore aggiunto che si forma nell'ambito della rete di relazioni cooperative, cioè sulla differenza tra il valore della produzione realizzata ed il costo dei fattori produttivi acquisiti esternamente alla rete.

Il suddetto valore, creato internamente alla rete, è destinato a fornire un'adeguata remunerazione ai partner di fornitura, ai lavoratori, ai finanzia-

1 "Un bene o un servizio vale non solo in quanto è stato prodotto e quindi incorpora delle utilità, ma perché qualcuno lo richiede e utilizzandolo soddisfa, più o meno adeguatamente, un proprio bisogno. Il valore proposto diventa riconosciuto se è accettato" (Mussari, 2011, pp. 75-76). Il "riconoscimento" dunque è legato all'accettazione del prezzo di mercato da parte del consumatore. Se manca il mercato, il "riconoscimento" è necessariamente "politico" (Farneti, 2004, p. 16). Pertanto, "i valori economici positivi riconducibili alla produzione per il consumo non sono ricavi, ma proventi di natura tributaria (imposte, trasferimenti attivi e, in misura minore, tasse)" (Borgonovi, Mussari, 2011, p. 109). 
tori, alla comunità sociale (le componenti di imposizione fiscale e di liberalità) ed all'azienda in sé (ammortamenti e accantonamenti per rischi e per riserve di utili).

Si tratta, più precisamente, di una particolare configurazione di valore aggiunto espresso al lordo degli ammortamenti, degli oneri finanziari e dei costi per beni e servizi acquisiti all'interno della rete, tutti costi che possono, più propriamente, essere considerati dal lato della distribuzione del valore aggiunto:

1) gli oneri finanziari, in quanto rappresentano, assieme ai dividendi, "una remunerazione del capitale d'impresa, comunque questo venga reperito, e cioè sotto forma di capitale proprio o di credito" (Lai, 1990, p. 190);

2) le quote di ammortamento, in quanto esprimono la remunerazione del capitale investito nelle immobilizzazioni, ma anche, in senso più ampio, la remunerazione dell'azienda in sé, assieme agli utili non distribuiti e agli accantonamenti per rischi, generici e specifici;

3) i costi per servizi di lavoro acquisiti all'interno della rete, al fine di "apprezzare" pienamente il contributo fornito dall'azienda in termini di valore creato e valore distribuito, "a partire dai caratteri che qualificano i rapporti di aggregazione, tenendo conto, cioè, del grado di coinvolgimento del fornitore nel sistema economico-tecnico dell'impresa" (Lai, 1990, p. 175) $)^{2}$.

Il valore aggiunto, come sopra definito nell'ambito della rete di relazioni cooperative, fornisce una rappresentazione di sintesi del valore creato, alternativa rispetto all'Economic Value Added (EVA) e a tutte le altre configurazioni di reddito residuale (Giannetti, 2013, p. 109 e ss).

La scelta di esprimere il valore creato mediante risultati reddituali lordi, piuttosto che risultati netti, rafforza la visione del capitale come valore derivato rispetto al reddito (Zappa, 1937, p. 98), in termini di "capitale di funzionamento", ma anche come "valore unico risultante dalla capitalizzazione dei redditi futuri" (Zappa, 1937, p. 96), dunque di "capitale economico".

\section{I contributi accolti nel presente numero della rivista}

Il presente numero della rivista include articoli che si inseriscono, per le tematiche trattate, nel percorso sopra delineato. In particolare, dopo un primo

${ }^{2}$ Si può distinguere, a questo riguardo, il valore aggiunto diretto, cioè direttamente riferibile all'azienda che riceve i servizi (di lavoro individuale), dal valore aggiunto indiretto (riferito agli specifici fornitori di servizi), dal valore aggiunto indotto sul territorio di riferimento. 
articolo centrato sul riconoscimento del valore creato, negli articoli successivi viene prima affrontato il tema della crisi aziendale, la sua individuazione e la previsione delle possibili insolvenze, approfondendo successivamente il tema del superamento della crisi, creando condizioni di sviluppo sostenibile, e del ruolo svolto dai sistemi di misurazione e controllo dei costi e delle performance nei diversi contesti aziendali.

Nel primo articolo, predisposto da Federica Ricci assieme ad altri ricercatori delle Università di Cassino e Napoli Parthenope, vengono presentati i risultati di una ricerca sulle relazioni esistenti tra il valore espresso dal mercato finanziario e una particolare configurazione di valore aggiunto legato all'efficienza del capitale intellettuale.

Nel successivo articolo di Velia Cenciarelli, Marco Maria Mattei e Giulio Greco, dopo aver discusso sulla possibile integrazione dei modelli tradizionali di previsione delle insolvenze, basati tipicamente su indicatori di bilancio, con ulteriori variabili predittive, vengono indagate le relazioni esistenti tra la pressione competitiva e la previsione dell'insolvenza.

Usando un campione di imprese private italiane nel periodo 2007-2014, gli autori hanno accertato che un aumento della concorrenza sul mercato dei prodotti influisce sulla possibilità di insolvenza. Hanno rilevato, inoltre, che i modelli che includono una misura specifica di pressione competitiva siano più efficaci di quelli che includono il mero controllo per il settore di appartenenza dell'azienda. Come misura della pressione competitiva, hanno utilizzato la cosiddetta "fluidità" - il cambiamento dell'oggetto sociale - mediante l'analisi testuale della descrizione dell'attività svolta.

Le riflessioni proposte nell'articolo su come integrare il quadro degli indicatori di bilancio nella previsione delle insolvenze sono particolarmente interessanti e di attualità. Si condivide, a tale riguardo che l'integrazione possa essere efficacemente basata su dati qualitativi.

Negli articoli successivi, i temi del superamento della crisi e della creazione di valore, determinando condizioni di sviluppo sostenibile, vengono affrontati interpretando il ruolo dei sistemi di misurazione e controllo dei costi e delle performance.

Lino Cinquini, Cristina Campanale, Alessandro Marelli e altri ricercatori dell'Università Sant'Anna di Pisa presentano un'analisi esplorativa su un insieme di aziende manifatturiere operanti in Italia, per interpretare gli strumenti informativi utilizzati e i cambiamenti intrapresi o auspicati per supportare strategie di crescita del business orientate ai servizi. Le aziende analizzate sono quelle che si stanno spostando dalla pura manifattura a una combinazione di prodotti e servizi integrati. Vengono presi in considerazione, in 
particolare, i diversi orientamenti strategici di tali aziende nella prospettiva della creazione di valore: differenziando i servizi resi, rafforzando i rapporti con i clienti, rendendo più ardua la competizione, determinando una migliore sostenibilità del business, prestando maggiore attenzione alla dimensione immateriale, sfruttando anche le nuove tecnologie digitali, quali Cloud, Big Data e Internet of Things.

Sempre interpretando il ruolo dei sistemi di misurazione e controllo, i successivi articoli del presente fascicolo sono centrati sulle aziende e amministrazioni pubbliche: Amministrazioni Centrali dello Stato; Università; Enti Locali.

Il tema della crisi e del cambiamento delle istituzioni di contabilità pubblica nel caso delle amministrazioni centrali dello Stato è trattato da Vania Palmieri e Mario Turco, a partire dalle seguenti domande di ricerca: Quali sono (se esistono) gli effetti generati dalla prima grande crisi del XXI secolo sulle istituzioni italiane di finanza e contabilità pubblica delle amministrazioni centrali dello Stato? È ad essa che sono ascrivibili i rilevanti cambiamenti di tali istituzioni nel periodo di riferimento? Si tratta di cambiamenti bruschi ed improvvisi o di cambiamenti graduali?

Per dare risposta alle suddette domande, gli autori si sono basati sull'utilizzo di un metodo qualitativo dato dall'analisi dei testi normativi e dei report ufficiali di commento ad essi, nonché dall'esperienza da "osservatore privilegiato" di uno dei coautori.

Tra i cambiamenti rilevanti adottati dalle amministrazioni pubbliche rientrano: 1) la sostituzione di budget in cui le spese sono dettagliate per singole voci con budget che lascino ai manager maggiore libertà nello scegliere il mix di risorse per raggiungere gli obiettivi; 2) l'adozione della base contabile economico-patrimoniale e di standard contabili simili a quelli applicati nel settore privato.

Di questo secondo cambiamento, con specifico riferimento agli atenei italiani, si sono occupati Giovanni Coran e Ugo Sostero, interpretando l'utilizzo delle nuove informazioni "sia all'interno del sistema aziendale (come strumento di monitoraggio delle condizioni di equilibrio e come supporto del processo decisionale) sia a beneficio dei soggetti esterni, migliorando il livello di accountability nei confronti dei vari stakeholder".

Come efficacemente evidenziano gli autori, tuttavia, "la comprensione e l'utilizzo di queste nuove informazioni sono talvolta ostacolati da alcune difficoltà di rappresentazione delle specificità gestionali degli atenei e dalle scelte che il legislatore $\mathrm{h}$ operato nel definire i criteri di esposizione delle poste di bilancio e degli schemi di rendicontazione". 
Gli schemi di analisi e rappresentazione delle informazioni di bilancio proposti da Coran e Sostero sono senz'altro di grande interesse sia per gli organi di governo sia per gli stakeholder sia per il mondo della ricerca. Tra le varie proposte, si sottolineano quelle relative alla rappresentazione della Posizione Finanziaria Netta (spesso a credito) e del capitale investito netto (spesso negativo), tenendo conto delle specifiche caratteristiche delle attività degli atenei, con particolare riguardo alle rilevanti entrate anticipate per tasse, contributi studenteschi, borse e contratti pluriennali che generano risconti passivi spesso superiori al capitale investito lordo.

L'ultimo articolo del fascicolo riguarda si occupa della rilevanza pratica dei sistemi di misurazione delle performance negli Enti Locali. Gli autori, Eleonora Cardillo, Carmela Rizza e Daniela Ruggeri affrontano, attraverso l'analisi in profondità di un caso aziendale, gli aspetti caratterizzanti la misurazione delle performance negli Enti Locali, individuando un framework teorico da impiegare per l'interpretazione della rilevanza pratica dei modelli proposti negli studi di Management Accounting, considerata anche la possibile diversa interpretazione fornita dagli organi politici e dagli organi tecnici. L'attenzione ai processi strategici ed istituzionali da parte dei politici e la gestione dei processi interni da parte dei tecnici possono determinare una differente interpretazione, ma anche una divergenza tra i modelli teorici e le applicazioni di tali modelli nelle realtà aziendali.

\section{References}

Bertini U. (1990), Il sistema d'azienda. Uno schema di analisi, Torino, Giappichelli.

Borgonovi E., Mussari R. (2011), Pubblico e privato: armonizzare gli opposti, Azienda Pubblica, 2.

Catturi G. (1990), Produrre e consumare, ma come? Verso l'ecologia aziendale, Padova, CEDAM.

Catturi G. (1997), Lezioni di Economia Aziendale, Padova, CEDAM.

Coda V. (2012), Il bene dell'impresa, in AA:VV, Valori d'impresa in azione, Milano, EGEA.

D’Onza G. (2008), Il sistema di controllo interno nella prospettiva del risk management, Milano, Giuffrè.

Farneti G. (2004), Ragioneria Pubblica, Milano, FrancoAngeli.

Freeman R.E., Rusconi G., Dorigatti M. (a cura di) (2007), Teoria degli stakeholder, Milano, FrancoAngeli.

Friedman M. (1970), The Social Responsibility of Business is to increase its profits, New York Times Magazine, 13 September.

Garzella S. (2019), Governo delle sinergie e creazione di valore nella gestione strategica dell'azienda, Torino, Giappichelli.

Giannessi E. (1960), Le aziende di produzione originaria. Le aziende agricole, Pisa, Cursi. 
Giannetti R. (2013), Dal reddito al valore. Analisi degli indicatori di creazione di valore basati sul reddito residuale, Milano, Giuffrè.

Greco G., D’Onza G. (2020), Dal controllo alla creazione di valore, Management Control, 2, pp. 5-9. Doi: 10.3280/MACO2020-002001.

Greco G., Presti C., Marchi L. (2019), Nuove prospettive su governance, audit, risk e performance management, Torino, Giappichelli.

Guatri L. (1990), La valutazione delle aziende, Milano, Giuffrè.

Lai A. (1990), Le aggregazioni di imprese, Milano, FrancoAngeli.

Marasca S. (2010), Intangibles e creazione di valore. Fra relazioni causali e "legami mancanti”, in: Marchi L., Marasca S. (a cura di), Le risorse immateriali nell'economia delle aziende, vol. II: Profili di misurazione e comunicazione, Bologna, il Mulino.

Marchi L. (2014), L'evoluzione degli scenari e delle teorie economico-aziendali: uno sguardo al passato rivolto al futuro, in AA.VV., Il legame fra coesione territoriale, sviluppo locale e performance d'impresa, Milano, FrancoAngeli.

Marchi L. (2019), Governo delle aziende e creazione di valore: da una prospettiva finanziaria ad una prospettiva economico-sociale, Management Control, 1 pp. 5-16. Doi: 10.320/MACO2019-001001.

Marchi L., Paolini A. (2020), Il controllo della sostenibilità: una prospettiva integrate, in Scritti in onore di Antonio Matacena, Milano, FrancoAngeli.

Maris B. (2000), Lettera Aperta ai Guru dell'Economia, Firenze, Ponte alle Grazie.

Matacena A. (1984), Impresa e ambiente. Il bilancio sociale, Bologna, Clueb.

Matacena A. (1993), La responsabilità sociale: da vincolo ad obiettivo. Verso una ridefinizione del finalismo d'impresa, in AA.VV., Istituzioni di economia d'Azienda. Scritti in onore di Carlo Masini, tomo I, Milano, EGEA.

Paolini A. (2018), L'azienda, in Marchi L. (a cura di), Introduzione all'economia aziendale, Torino, Giappichelli.

Paolini A. (a cura di) (2018), Verso l'Economia circolare, Macerata, EUM.

Porter M.E. (1985), Competitive Advantage: creating and sustaining superior Performance, New York, Free Press.

Quagli A. (2009), Dal fair value al fairy value: coerenza concettuale e condizioni di impiego del fair value negli IFRS, Financial Reporting, 1.

Ronen J. (2008), To Fair Value or Not to Fair Value: a Broader Perspective, Abacus, 2.

Varanini F. (2020), Apprendere dal virus. Il contagio delle esperienze, Parole di Management, $30 / 08$. 